Journal of Computer Science 7 (5): 611-618, 2011

ISSN 1549-3636

(C) 2011 Science Publications

\title{
Weighted Kernel Density Estimation of the Prepulse Inhibition Test
}

\author{
${ }^{1}$ Hongbo Zhou, ${ }^{1}$ Qiang Cheng, ${ }^{2}$ Hong-Ju Yang and ${ }^{2}$ Haiyun Xu \\ ${ }^{1}$ Department of Computer Science, Southern Illinois University Carbondale, 62901, IL \\ ${ }^{2}$ Department of Anatomy, Southern Illinois University Carbondale, 62901, IL
}

\begin{abstract}
Problem statement: The goal of this study was to devise a more reliable and sensitive method for analysis of experimental data of the Prepulse Inhibition (PPI), the reduction in startle reaction towards a startle-eliciting "pulse" stimulus when it is shortly preceded by a sub-threshold "prepulse" stimulus. Approach: Different from the conventional simple averaging-based method, we proposed a probabilistic approach to modeling the PPI data. With this probabilistic description, we reconstructed complete response signals from the PPI data and devised a nonparametric weighted Kernel Density Estimation (KDE) method to tackle two important issues in PPI data related density estimation: instability and limited number of samples. We designed two sets of animal experiments using different medicines and compared the KDE based method with the conventional simpleaveraging based method. Results: Our results showed that the KDE method performed better than the conventional method and offered some advantages over the conventional method. Conclusion: The new method provided a more reliable and sensitive approach to the post-session analysis of PPI data.
\end{abstract}

Key words: Kernel density estimation, prepulse inhibitation test, startle response, Clozapine (CLZ), Quetiapine (QTP), non-parametric, random variables, Cuprizone (CPZ), dopamine hyperactivity

\section{INTRODUCTION}

Prepulse Inhibition (PPI) refers to the reduction in startle reaction towards a startle-eliciting "pulse" stimulus when it is shortly preceded by a subthreshold "prepulse" stimulus ((Fatemi, 2008; Xu et al., 2010); Fig. 1). This test provides an operational measure of sensory gating of subjects. Disruption of PPI has been related to central dopamine hyperactivity (Swerdlow et al., 1986; Fatemi, 2008) and is observed in schizophrenic patients (Bolino et al., 1994; Perry and Braff, 1994) and in animal models of this mental disorder (Bakshi et al., 1999; Pen and Moreau, 2002; Moy et al., 2006). In our recent studies we established a novel animal model of schizophrenia in C57BL/6 mice exposed to Cuprizone (CPZ), a copper chelator. The cuprizone-exposed mice show some behavioral changes reminiscent of certain schizophrenia symptoms. One of which is deficit in PPI (Xu et al., 2009; 2010). Furthermore, the PPI deficit can be prevented or attenuated by antipsychotic drugs including Clozapine (CLZ) and Quetiapine (QTP) (Xu et al., 2010).

In rodents (mouse and rat) the startle reaction in PPI test can be measured by recording whole body startle, which occurs following the presentation of an eliciting stimulus. The body startles are sensed by a piezoelectric sensor and read out as electrical currents changing as the function of time (Fig. 2). A typical PPI test includes 5 starting and 5 ending startle trials as well as 8-10 identical testing blocks. Each testing block consists of: a no-stimulus trial, a startle trial without the prepulse and three prepulse-startle trials each of which had a pre-pulse stimulus $(3,6$, or $12 \mathrm{~dB}$ above the background sound levels) prior to a startle stimulus (100 ms after the prepulse). These trials are ordered randomly and separated by variable inter-trial intervals to minimize habituation of the subjects to the sound stimuli across the trials.

A typical startle response, as shown in Fig. 2, can be described by several parameters including:

- Start $(\mathrm{mV})$ : The voltage at the start of the response window

- Vmax (mV): The highest voltage during the response window, or the "peak" of the response

- Tmax (ms): The time in milliseconds at which the Vmax appear after the start of the response window

- Avg. (mV): The averaged voltage across the entire response windo 


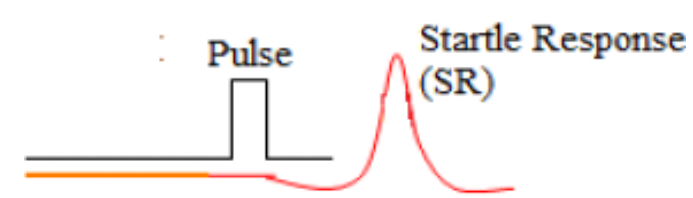

(a)

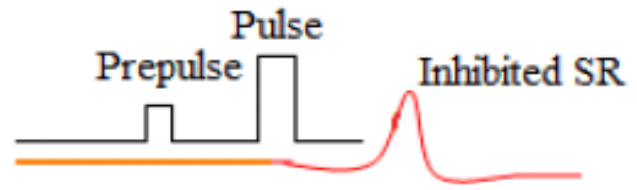

(b)

Fig. 1: The mechanic illustration of the prepulse inhibition test

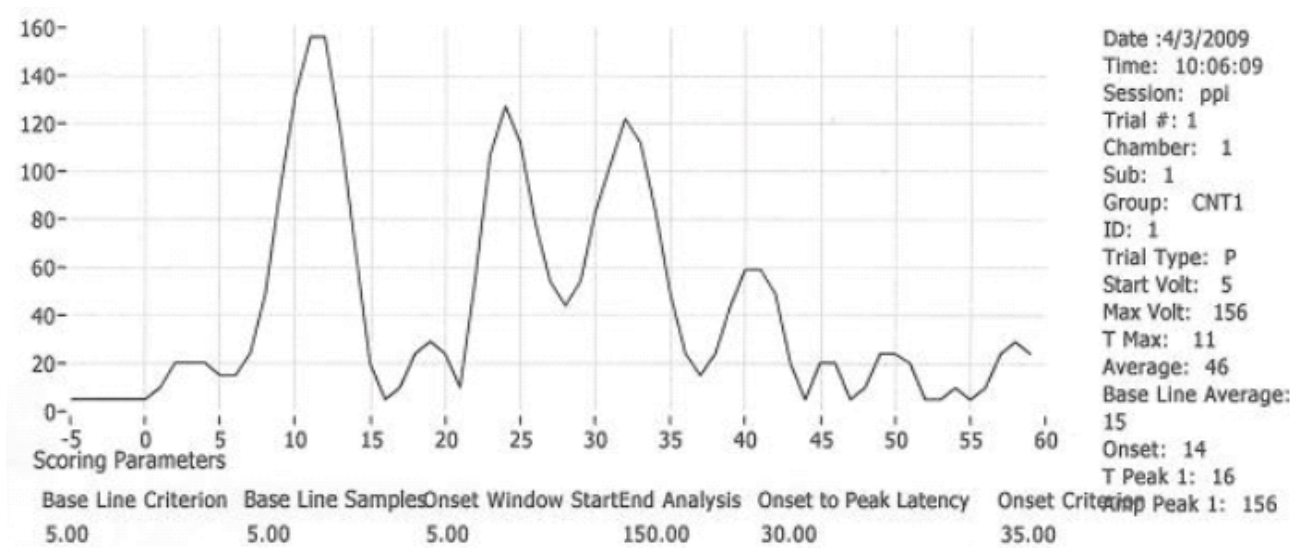

Fig. 2: A typical startle response graph. The horizontal axis is the number of record samples (ms). The vertical axis represents the amplitude of the startle response in millivolts

- Baseline $(\mathrm{mV})$ : The averaged voltage of five samples following the start of the response window. It informs the user if there is any excessive activity prior to the stimulus

- T-Peak 1 (ms): The time at which the first peak appears

- Amp P1 (mV): The amplitude of the first peak

These parameters produce considerable information in different aspects of a startle response. Based on this information researchers can determine if a startle response is caused by a sound stimulus or due to a random movement not-related to the stimulus. Only those stimulus-related startle responses should be used to calculate the PPI of the subject.

In a recent study we created a program for the postsession data analysis of the PPI test (Zhou et al., 2009). This program has the functions of (1) grouping data under different chambers and trials; (2) eliminating the questionable data and (3) performing batch processing for a number of files at one time. Therefore, it is a great helper in analyzing PPI data.

The goal of this study is to provide an alternative method for the analysis of PPI data. The new method is expected to be more reliable and sensitive than the existing conventional method. For the first time, we applied the Kernel Density Estimation (KDE) in the analysis of PPI data and compared this method with the conventional method. The results showed that the Kernel density estimation performed as well or better than the conventional method and offered a number of advantages.

Kernel density estimation: Kernel density estimation is a non-parametric method of estimating the probability density function of a random variable. Given some data about a sample of a population, KDE can extrapolate the data to the entire population (Everitt, 2006; Alfred et al., 2010). This method is widely used in inferring population statistics based on limited, noisy samples of continuous random variables. In previous studies, KDE was used to improve the histogram of laboratory data (Willard and Connelly, 1992) and was applied for the analysis of polymorphic variation in drug metabolism (Herman and Laverty, 1994).

Given a set of independent samples $\left\{S_{i}\right\}_{i=1}^{n}$ for the random variable, KDE can construct the probability density function $\mathrm{f}(\mathrm{r})$ by the following procedures:

Step 1: Choose a kernel function $\mathrm{k}\left(\mathrm{s}_{\mathrm{i}}\right)$ for each sample $\mathrm{s}_{\mathrm{i}}$ Step 2: Construct $\mathrm{f}$ ( $\mathrm{r}$ ) by adding all kernel functions together and thus have the formula (1):

$$
\mathrm{f}(\mathrm{r})=\frac{1}{\mathrm{n}} \sum_{\mathrm{n}=1}^{\mathrm{n}} \mathrm{k}\left(\mathrm{s}_{\mathrm{i}}\right)
$$




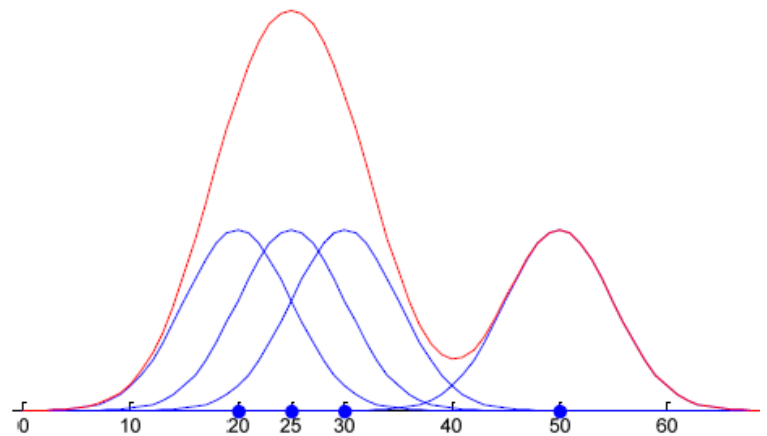

Fig. 3: A typical KDE with optimal smoothness. We had four samples at 20, 25, 30 and 50 respectively (the blue points). For each of them we created a Gaussian kernel function (the blue curves) with a variance of 5 . Then, $f(r)$ (the red curve) was constructed by linearly combining all kernel functions together

An example of KDE using the above procedures was shown in Fig. 3.

Kernel density estimation may be used in post session analysis of PPI test: In PPI test, the variation for each animal and across a group of animals is usually in a wide range. Also, the system may produce noises, incomplete and unstable data, even errors. Therefore, post-session analysis of PPI data is a challenging issue. In this study, we modeled the PPI data probabilistically and used the KDE method to deal with the noises and outliers.

According to its definition, the PPI of each animal is calculated by the formula (2):

$$
\text { PPI }=\frac{\left(\mathrm{V}_{\max } \text { without prepulse }-\mathrm{V}_{\max } \text { with a prepulse }\right)}{\mathrm{V} \text { max without prepulse }} \times 100
$$

We denote the $\mathrm{V}_{\max }$ without prepulse as $\mathrm{AV}(\mathrm{P})$ and those with a prepulse as $\mathrm{AV}\left(\mathrm{PP}_{1}\right), \operatorname{AV}\left(\mathrm{PP}_{2}\right)$, or $\mathrm{AV}\left(\mathrm{PP}_{3}\right)$, respectively. Then, the following formulas will be used:

$$
\left\{\begin{array}{l}
\mathrm{PPI}_{1}=\frac{\mathrm{AV}(\mathrm{P})-\mathrm{AV}\left(\mathrm{PP}_{1}\right)}{\mathrm{AV}(\mathrm{P})} \times 100 \% \\
\mathrm{PPI}_{2}=\frac{\mathrm{AV}(\mathrm{P})-\mathrm{AV}\left(\mathrm{PP}_{2}\right)}{\mathrm{AV}(\mathrm{P})} \times 100 \% \\
\mathrm{PPI}_{3}=\frac{\mathrm{AV}(\mathrm{P})-\mathrm{AV}\left(\mathrm{PP}_{3}\right)}{\mathrm{AV}(\mathrm{P})} \times 100 \%
\end{array}\right.
$$

The reconstruction of startle response using Gaussian kernel windows: Different from the simple averaging method, we proposed a probabilistic approach to modeling the PPI data. Specifically, we regard the startle response $\mathrm{V}_{\max }$ as a random variable $\mathrm{SR}$ and estimate the PPI based on "the most probable" values of the startle response for each group. The startle responses from an experiment are taken as independent samples from a true underlying distribution that emits the random variable $\mathrm{SR}$. We denote these sampled examples as $\left\{\mathrm{SR}_{1}, . ., \mathrm{SR}_{\mathrm{i}}, \ldots, \mathrm{SR}_{\mathrm{n}}\right\}$, where $\mathrm{I}=1,2, \ldots, \mathrm{n}$ and $\mathrm{n}$ is the total number of sampled examples.

To determine the "most probable" value for SR based on these limited samples, we used the KDE (also known as the Parzen window) method to estimate the probability density function $\mathrm{f}(\mathrm{SR})$, which is defined as:

$$
f(S R)=\frac{1}{n}-\sum_{i=1}^{n} \frac{1}{h_{i}} K\left(\frac{S R-S R_{i}}{h_{i}}\right)
$$

where, $\mathrm{k}$ is a kernel function and $\mathrm{h}_{\mathrm{i}}, \mathrm{i}=1, \ldots, \mathrm{n}$ are bandwidths. Some popular choices for the kernel function $\mathrm{K}$ include Gaussian, Epanechnikov, exponential, cosine kernels.

An important practical issue in using the above equation is how to determine a reasonable bandwidth $h_{i}$ for the corresponding data point $\mathrm{x}_{\mathrm{i}}$. If the bandwidth is too small, the resulted estimation would be undersmoothed or irregular; on the other hand, large $h_{i}$ would produce an over-smoothed probability distribution. We chose $h_{i}$ to be the range of the meaningful startle responses in this study.

By using the proposed method, we actually reconstructed the effective responses over the whole time durations. Although this reconstruction only used the $\mathrm{V}_{\max }$, we can obtain estimates at a point prior to the starting time. Hence, the missing information before the starting time of the records will be recovered. An illustration of this reconstruction is given in Fig. 4.

Stabilizing the kernel density estimation: Having chosen the kernel function and its bandwidth, we are in a position to estimate the density distribution of the population based on finite samples of $S R$. For PPI data analysis, two important issues remain to be addressed. (1) The instability as mentioned before. Due to the instability, the variance of the population may become large and it will directly affect the range of the population response and thus the bandwidth for Gaussian kernel functions. This will lead to deteriorated results of the KDE. (2) The limited number of samples. If the number of the samples is small, it will require other restrictions in interpreting the data and thus lead to a less valid estimation. Ideally, increasing the population size should overcome the above two difficulties. However, this is often in conflict with the limited uses of animals in laboratory experiments. 


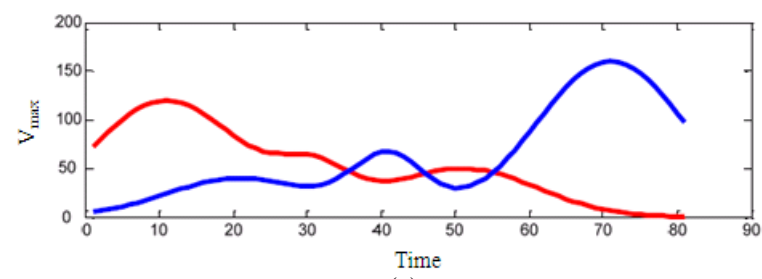

(a)

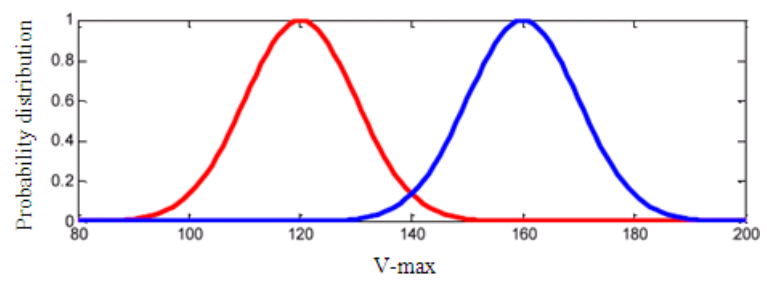

(b)

Fig. 4: Illustration of the reconstruction of startle response using Gaussian kernel windows. (a) shows two simulated startle response curves: the red one with a $\mathrm{V}_{\max }$ of 120 attained at 11th milisecond; and the blue one with a $\mathrm{V}_{\max }$ of 160 attained at $71 \mathrm{st}$ mili-second. (b) shows the reconstructed startle response using a Gaussian kernel with a bandwidth of $60 \mathrm{mv}$. The Xcoordinate of (b) is the $\mathrm{V}_{\max }(\mathrm{mv})$ and $\mathrm{Y}$ coordinate is the probability density.

Toward computationally addressing these two issues, we assumed that the meaningful startle response of a mouse varies around the population mean. And we introduced a weight $\mathrm{W}\left(\mathrm{SR}_{\mathrm{i}}\right)$ for each $\mathrm{SR}_{\mathrm{i}}$ to stabilize the estimation:

$$
\mathrm{W}\left(\mathrm{SR}_{\mathrm{i}}\right)=\mathrm{e}^{\overline{1\left(1-\mid \mathrm{SR}_{\mathrm{i}}-\left\langle\overline{\mathrm{SR}_{\mathrm{i}}}\right\}\right)}}
$$

where, $\left\{\mathrm{SR}_{\mathrm{i}}\right\}$ is the set of all sampled examples within a group and $\left\{\mathrm{SR}_{\mathrm{i}}\right\}$ is the population mean, max $\left(\left\{\mathrm{SR}_{\mathrm{i}}\right\}\right)$ is the maximum of all $\mathrm{SR}_{\mathrm{i}}$. Here $\mathrm{p}$ is a scaling factor. In this study, we set $p=0.1$ in all experiments. With this weighting scheme, the kernel functions near the population mean can get a large weight compared to those far away from the population mean.

Therefore, we now have a weighted $\mathrm{KDE} f(\mathrm{SR})_{\mathrm{w}}$ as follows:

$$
\mathrm{f}\left(\mathrm{SR}_{\mathrm{i}}\right)_{\mathrm{w}}=\frac{1}{\mathrm{n}} \sum_{\mathrm{i}=1}^{\mathrm{n}} \frac{1}{\mathrm{~h}_{\mathrm{i}}} \mathrm{W}\left(\mathrm{SR}_{\mathrm{i}}\right) * \mathrm{~K}\left(\frac{\mathrm{SR}-\mathrm{SR}_{\mathrm{i}}}{\mathrm{h}_{\mathrm{i}}}\right)
$$

\section{MATERIALS AND METHODS}

Implementation of PPI test: As described in previous studies (Xu et al., 2009; 2010), each mouse was placed into a small Plexiglas cylinder within a large soundattenuating chamber (San Diego Instruments, CA, USA). The cylinder was seated upon a piezoelectric transducer, which allows vibrations to be quantified and displayed on a computer. The background sound levels (74-75 dB) and calibration of the acoustic stimuli were confirmed with a digital sound level meter. After a 5min habituation period, PPI test sessions were conducted. Each session started and ended with five startle trials (40 ms; $120 \mathrm{~dB}$ ), respectively. Between the starting and ending startle trials, there were eight identical blocks consisting of the following five trials: a no-stimulus trial, a startle trial and three prepulse-startle trials, each of which had a pre-pulse stimulus (3, 6, or $12 \mathrm{~dB}$ above the background sound levels) prior to a startle stimulus (100 ms after the prepulse). The average inter-trial interval was $15 \mathrm{~s}$. Measures were taken of the startle amplitude for each trial, defined as the peak response during a 65-ms sampling window starting from the onset of a startle stimulus.

Experimental animal groups: Two independent experiments were performed using male C57BL/6 mice (6-weeks old, 20 to 22 g) purchased from Charles River Laboratories (Wilmington, MA, USA). After an acclimatization period of 10 days, the C57BL/6 mice were randomly assigned to any one of the following four groups (8-12 mice/group) for the first experiment: The CNT group, in which mice ate the standard diet without CPZ; the CPZ group, in which mice ate the diet mixed with CPZ (0.2\% by weight); the CLZ group, in which mice received CLZ (10 $\mathrm{mg} \mathrm{kg}^{-1}$ day $^{-1}$ ) via intraperitoneal injection (i.p.); and the CPZ+CLZ group, in which mice received CPZ and CLZ. The treatment continued for 21 days. On the 22nd day, all mice were subjected to PPI test as described above. The second experiment was designed in the same way as the first one and consisted of the four groups of CNT, CPZ, QTP and CPZ+QTP. QTP was also administered via i.p. at the dose of $10 \mathrm{mg} \mathrm{kg}^{-1}$ day $^{-1}$. The treatments continued for 21 days. On the 22nd day, all mice were subjected to PPI test as described above.

All animal procedures in this study were in accordance with the National Institute of Health Guide for the Care and Use of Laboratory Animals and were approved by the Animal Care and Use Committee of Southern Illinois University Carbondale.

\section{RESULTS}

Post-session analysis of PPI data by the kernel density estimation: First, we used the no-stimulus trials to calibrate the KDE method. The no-stimulus trials reflect the rest state of mouse restricted in the 
cylinder under the background sound level. All mice are expected to have same levels of rest state according to the neural mechanism of PPI. In accordance with the expectation, we found that all four animal groups in the first experiment have an identical density estimation of Vmax as shown in Fig. 5. The second experiment showed the same results (not shown). These results suggest that the system was stable and the KDE method may be used as an alternative method for post-session analysis of PPI data. In addition, the choice for the bandwidth of the kernel function here was justified well as it resulted in a complete overlap of all four density estimation curves into one curve.

Then, we proceeded to perform the density estimation for each type of trials. For the startle trials, our results (Fig. 6) showed that only the CPZ group (see blue line) had a smaller startle response in response to the same pulse $(\mathrm{P})$ whereas the other three groups had overlapped density estimations. This is in accordance with the mathematical expectation based on the computing formula of PPI as PPI is in direct proportion to the $\mathrm{V}_{\max }$ of the startle trials. "The most probable" Vmax of all four groups were also shown in this Fig. 6. For the second experiment, we got similar results (not shown).

For the first experiment, Fig. 7-9 showed the density estimation for the other three groups PP1, PP2, PP3 respectively. For the second experiment, we used similar estimations for each group (not shown).

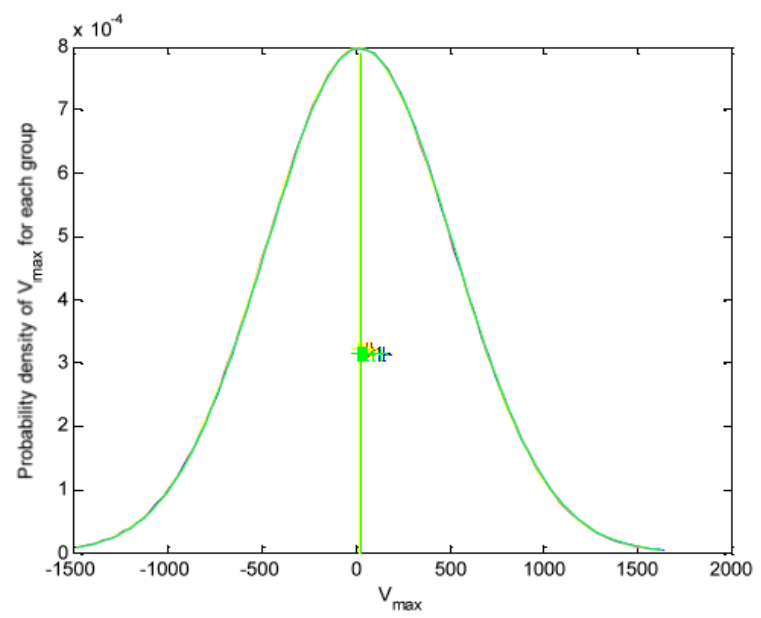

Fig. 5: Kernel density estimation of no-stimulus trials in the first experiment. The green, blue, red and yellow curves represent groups CNT, CPZ, CLZ and CPZ+CLZ, respectively. The dots around $\mathrm{y}$ $=3 \times 10^{-4}$ are the real distributions of the samples. All four groups overlap completely and attain a maximum of probability around $\mathrm{V}_{\max }=$ $24 \mathrm{mv}$

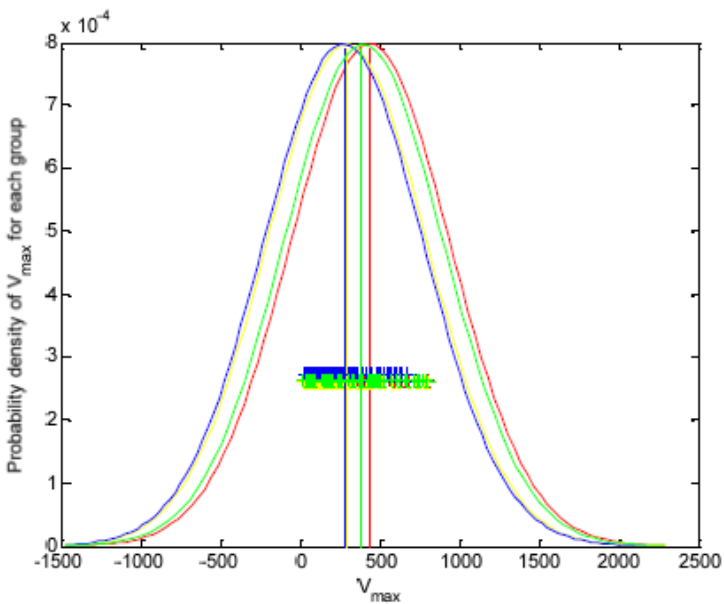

Fig. 6: Kernel density estimation for the startle trials (P) of the first experiment. The green, blue, red and yellow curves represent groups CNT, CPZ, CLZ and CPZ+CLZ, respectively. The dots around $y$ $=3 \times 10^{-4}$ are the real distributions of the samples. All four groups had their corresponding maximum of probability as shown by the four color lines. The maximum of probability for each group CLZ, CPZ+CLZ, CPZ and CNT is attained when Vmax is 431, 290, 278 and $379 \mathrm{mv}$, respectively.

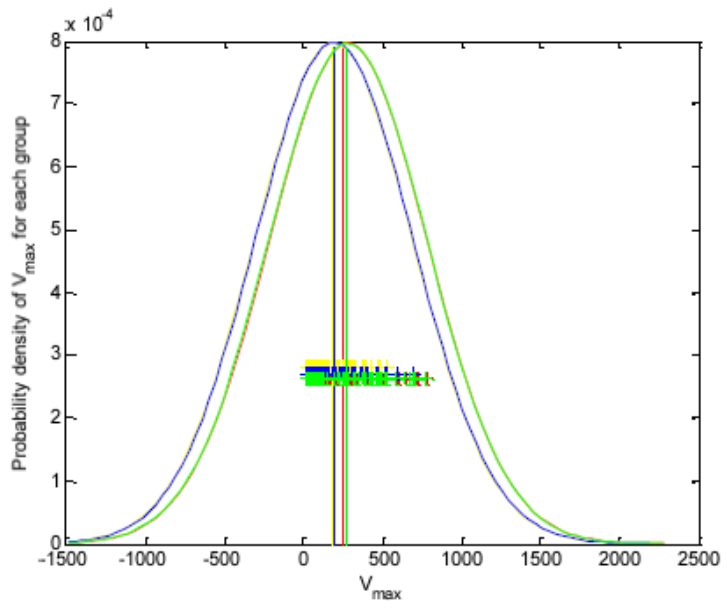

Fig. 7: Kernel density estimation for the PP1 trials of the first experiment. The green, blue, red and yellow curves represent groups CNT, CPZ, CLZ and $\mathrm{CPZ}+\mathrm{CLZ}$, respectively. The dots around $\mathrm{y}$ $=3 \times 10^{-4}$ are the real distributions of the samples. All four groups had their corresponding maximum of probability as shown by the four color lines. The maximum of probability for each group CLZ, CPZ+CLZ, CPZ and CNT is attained when $\mathrm{V}_{\max }$ is 260, 205, 204 and $259 \mathrm{mv}$, respectively 


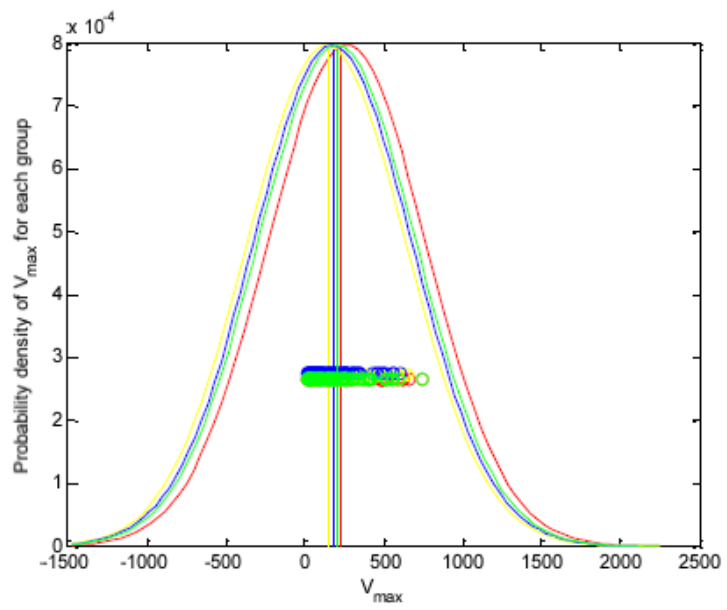

Fig. 8: Kernel density estimation for the PP2 trials of the first experiment. The green, blue, red and yellow curves represent groups CNT, CPZ, CLZ and CPZ+CLZ, respectively. The dots around y $=3 \times 10^{-4}$ are the real distributions of the samples. All four groups had their corresponding maximum of probability as shown by the four color lines. The maximum of probability for each group CLZ, CPZ+CLZ, CPZ and CNT is attained when $\mathrm{V}_{\max }$ is 247,128 , 168 and $198 \mathrm{mv}$, respectively

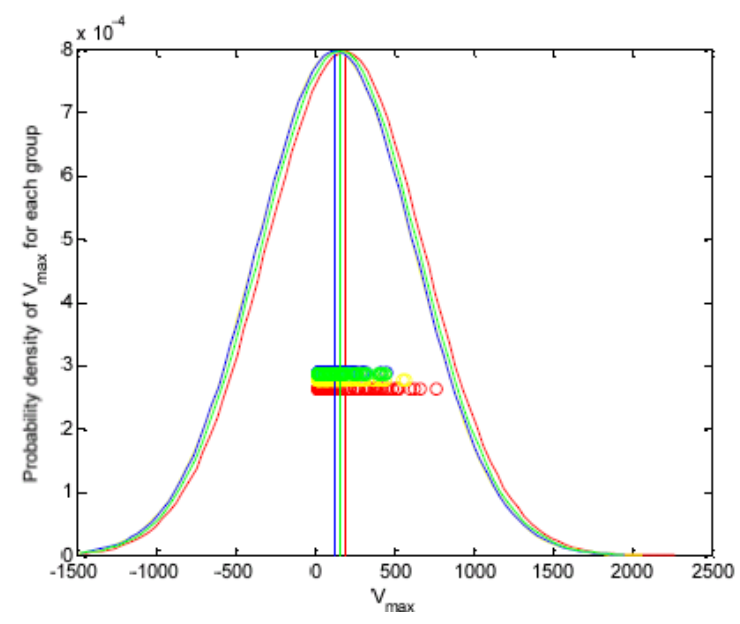

Fig. 9: Kernel density estimation for the PP3 trials of the first experiment. The green, blue, red and yellow curves represent groups CNT, CPZ, CLZ and CPZ+CLZ, respectively. The dots around y $=3 \times 10^{-4}$ are the real distributions of the samples. All four groups had their corresponding maximum of probability as shown by the four color lines. The maximum of probability for each group CLZ, CPZ+CLZ, CPZ and CNT is attained when $V_{\text {max }}$ is 196,133 , 136 and $157 \mathrm{mv}$, respectively

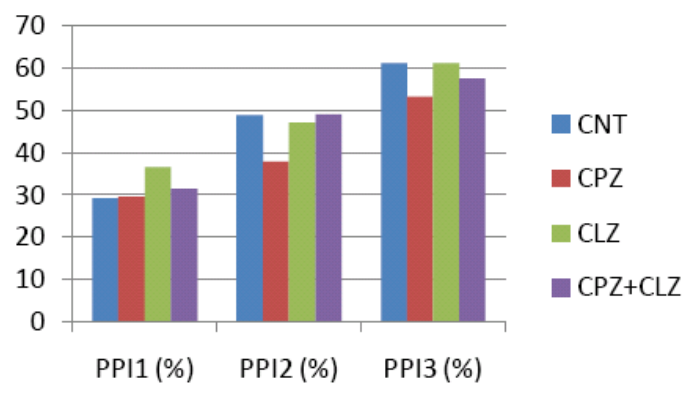

Fig. 10: PPIs of different animal groups in the first experiment computed by the traditional method. Abbreviations: CNT, controls; CPZ, cuprizone; CLZ, clozapine

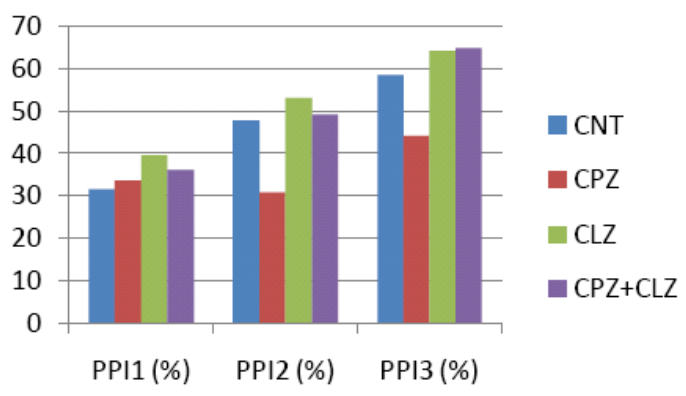

Fig. 11: PPIs of different animal groups in the first experiment computed by the KDE method. Abbreviations: CNT, controls; CPZ, cuprizone; CLZ, clozapine.

Table 1: Comparing the PPIs of different animal groups in the first experiment. Graphical illustration of the results for each method is shown in Fig. 10 and 11, respectively

\begin{tabular}{lllll}
\hline & Groups & $\mathrm{PPI}_{1}(\%)$ & $\mathrm{PPI}_{2}(\%)$ & $\mathrm{PPI}_{3}(\%)$ \\
\hline Conventional & CNT & 29.16 & 48.81 & 61.06 \\
method & CPZ & 29.57 & 37.79 & 53.28 \\
& CLZ & 36.43 & 47.10 & 61.22 \\
Score: 142.358 & CPZ+CLZ & 31.38 & 48.92 & 57.63 \\
KDE & & & & \\
& CNT & 31.52 & 47.82 & 58.51 \\
& CPZ & 33.63 & 30.85 & 44.13 \\
& CLZ & 39.59 & 53.17 & 64.29 \\
Score: 1044.129 & CPZ+CLZ & 36.23 & 49.13 & 64.87
\end{tabular}

Abbreviations: CNT, controls; CPZ, cuprizone; CLZ, clozapine

Finally, the PPIs of each animal group were computed by the conventional method and KDE using the same formulas (3). All computed results were summarized in Table 1 (for the first experiment) and Table 2 (for the second experiment), in which The score was defined as the sum of distances between the CPZ group and all other three groups as only the $\mathrm{CPZ}$ group was a distinct group with the smallest PPIs. 


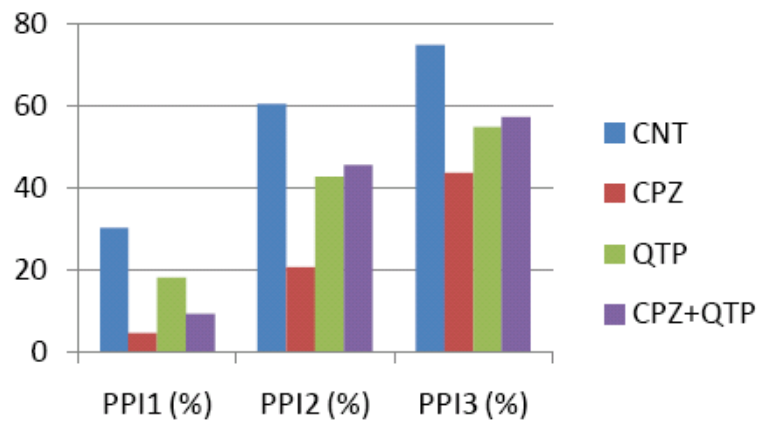

Fig. 12: PPIs of different animal groups in the second experiment computed by the traditional method. Abbreviations: CNT, controls; CPZ, cuprizone; QTP, quetiapine.

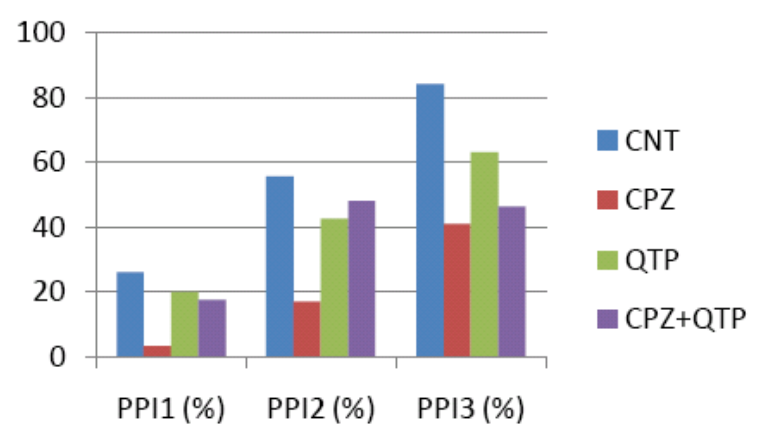

Fig. 13: PPIs of different animal groups in the second experiment computed by the KDE method. Abbreviations: CNT, controls; CPZ, cuprizone; QTP, quetiapine.

Table 2: Comparing the PPIs of different animal groups in the second experiment. Graphical illustration of the results for each method is shown in Fig. 12 and 13, respectively

\begin{tabular}{llrll}
\hline & Groups & $\mathrm{PPI}_{1}(\%)$ & $\mathrm{PPI}_{2}(\%)$ & $\mathrm{PPI}_{3}(\%)$ \\
\hline Conventional & CNT & 30.12 & 60.44 & 74.88 \\
method & CPZ & 4.46 & 20.53 & 43.58 \\
& QTP & 18.03 & 42.79 & 54.73 \\
Score: 1292.747 & & 9.25 & 45.66 & 57.33 \\
KDE & CNT & 26.06 & 55.65 & 84.23 \\
& CPZ & 3.36 & 16.94 & 40.96 \\
& QTP & 19.98 & 42.57 & 63.08 \\
Score: 2388.982 & CPZ+QTP & 17.36 & 48.09 & 46.19
\end{tabular}

Abbreviations: CNT, controls; CPZ, cuprizone; QTP, quetiapine.

Although the results from the two methods suggest the same conclusion that mice in the CPZ group had lower PPI than the other three groups, the KDE method showed some advantages over the conventional method. These advantages and their significance will be discussed in the discussion part.

\section{DISCUSSION}

Compared to the conventional method, the proposed weighted KDE method has the following advantages in the analysis of PPI data.

First, the weighted KDE performed better than the conventional method. This better performance was objectively evaluated by the values of score resulted from the PPI of all groups computed using the two methods. According to the definition above, the value of a score is larger the performance of the method is better from which the score is computed. It is clear that the weighted KDE method produced larger scores in both the first and second experiments as shown in Table 1 and 2.

In Table 1, the weighted KDE method enlarged the differences between the CPZ group and the other three groups in PPI2 and PPI3; therefore it produced a larger score compared to the conventional method. In other words, the weighted KDE method increases the sensitivity of the PPI test thus can diminish the demand of animals required for an experiment. Obviously, this is an animal-friendly advantage. Also, it can decrease the workload for lab researchers.

Second, the weighted KDE method appears more robust to noise and subject variability. The weighted KDE method reconstructs the startle responses from a sample of examples. The reconstructed responses are relatively insensitive to the noise and subject variability. As seen from Fig. 6-9, the weighted KDE can give the most probable values of the estimation, which correspond to the modes of the probability density functions. The estimated density functions are symmetric around the mode. We have used the mode values in calculating the PPIs. The results appear more stable than conventional method. The stabilizing technique with weighting has further enhanced the stability. The choice of bandwidth used in the weighted KDE appears natural and has a meaningful interpretation.

\section{CONCLUSION}

As a conclusion, we shall recommend the probabilistic approaches on similar studies. As shown in this work, the probabilistic modeling is quite effective in considering incomplete and unstable response signals, which are vital to the analysis of signals generated from animal behavioral experiments. Also, the weighting processing in this study is quite important since the number of samples are limited and there might be outliers in the measured signals. 
The next line of research will include investigating whether other factors than Vmax can be also significant in determining the effect of PPI and a possible further enhancement of the sensitivity of the PPI test.

\section{ACKNOWLEDGMENT}

All data came from the animal studies, which were performed in the lab of HX and supported by a start up fund (School of Medicine) and a faculty seed grant of Southern Illinois University Carbondale to HX. This work is also partly supported by a NSF grant CNS0855221 to QC.

\section{REFERENCES}

Alfred, R., A.H. Ahmad, J. Payne, C. William and L. Ambu, 2010. Density and Population Estimation of the Bornean Elephants (Elephas maximus borneensis) in Sabah. OnLine J. Biol. Sci., 10: 92102. DOI: $10.3844 /$ ojbsci.2010.92.102

Bakshi, V.P., M. Tricklebank, H.C. Neijt, V. LehmannMasten and M.A. Geyer, 1999. Disruption of prepulse inhibition and increases in locomotor activity by competitive N-methyl-D-aspartate receptor antagonists in rats. JPET, 288: 643-652. ISSN: 0022-3565

Bolino, F., V.D. Michele, L.D. Cicco, V. Manna and E. Daneluzzo et al., 1994. Sensorimotor gating and habituation evoked by electro-cutaneous stimulation in schizophrenia. Biol. Psychiatry, 36: 670-679. PII: 0006-3223(94)91176-2

Everitt, B.S., 2006. The Cambridge Dictionary of Statistics. 3rd Edn., Cambridge University Press, Cambridge, ISBN-10: 0521690277, pp: 444.

Fatemi, S.H., 2008. The role of neurodevelopmental genes in infectious etiology of autism. Am. J. Biochem. Biotechnol., 4: 177-182. DOI: 10.3844/ajbbsp.2008.177.182

Herman, R.J. and W.H. Laverty, 1994. Analysis of polymorphic variation in drug metabolism: I. Kernal density estimation. Clin. Invest. Med., 17: 281-189. PMID: 7982291
Moy, S.S., A. Perez, B.H. Koller and G.E. Duncan, 2006. Amphetamine- induced disruption of prepulse inhibition in mice with reduced NMDA receptor function. Brain Res., 1089: 186-194. DOI: 10.1016/J.BRAINRES.2006.03.073

Pen, G.L. and J.L. Moreau, 2002. Disruption of prepulse inhibition of startle reflex in a neurodevelopmental model of schizophrenia: Reversal by clozapine, olanzapine and risperidone but not by haloperidol. Neuropsychopharmacology, 27: 1-11. DOI: 10.1016/S0893-133x(01)00383-9

Perry, W. and D.L. Braff, 1994. Information-processing deficits and thought disorder in schizophrenia. Am. J. Psychiatry, 151: 363-367. ISSN: 0002-953X

Swerdlow, N.R., D.L. Braff, M.A. Geyer and G.F. Koob, 1986. Central dopamine hyperactivity in rats mimics abnormal acoustic startle response in schizophrenics. Biol. Psychiatry, 21: 23-33. PII: 0006-3223(86)90005-3

Willard, K.E. and D.P. Connelly, 1992. Nonparametric probability density estimation: Improvements to the histogram for laboratory data. Comput. Biomed. Res., 25: 17-28. DOI: 10.1016/00104809(92)90032-6

$\mathrm{Xu}, \mathrm{H} ., \mathrm{H}$. Yang, Y. Zhang, R. Clough and R. Browning et al., 2009. Behavioral and neurobiological changes in C57BL/6 Mice exposed to cuprizone. Behav. Neurosci., 123: 418-429. DOI: 10.1037/a0014477

$\mathrm{Xu}, \mathrm{H} .$, H.J. Yang, B. McConomy, R. Browning and X.M. Li, 2010. Behavioral and neurobiological changes in C57BL/6 mouse exposed to cuprizone: Effects of antipsychotics. Front Behav. Neurosci., 4: 1-8. DOI: 10.3389/fnbeh.2010.00008

Zhou, H., H.-J. Yang, H. Xu and Q. Cheng, 2009. A new computational tool for the post session analysis of the prepulse inhibition test in neural science. Proceedings of the 2009 International Conference on Computational Science and Engineering, Aug. 29-31, Vancouver, Canada, pp: 1077-1080.

http://doi.ieeecomputersociety.org/10.1109/CSE.20 09.61 